\title{
Effects of Cognitive Behavioral Therapy on Daily Living Skills in Children with High-Functioning Autism and Concurrent Anxiety Disorders
}

\author{
Amy Drahota $\cdot$ Jeffrey J. Wood $\cdot$ Karen M. Sze $\cdot$ \\ Marilyn Van Dyke
}

Published online: 28 May 2010

(C) The Author(s) 2010. This article is published with open access at Springerlink.com

\begin{abstract}
CBT is a promising treatment for children with autism spectrum disorders (ASD) and focuses, in part, on children's independence and self-help skills. In a trial of CBT for anxiety in ASD (Wood et al. in J Child Psychol Psychiatry 50:224-234, 2009), children's daily living skills and related parental intrusiveness were assessed. Forty children with ASD (7-11 years) and their primary caregiver were randomly assigned to an immediate treatment (IT; $n=17$ ) or 3 -month waitlist (WL; $n=23$ ) condition. In comparison to WL, IT parents reported increases in children's total and personal daily living skills, and reduced involvement in their children's private daily routines. Reductions correlated with reduced anxiety severity. These results provide preliminary evidence that CBT may yield increased independence and daily living skills among children with ASD.
\end{abstract}

Keywords Cognitive behavioral therapy .

Autism spectrum disorders · Daily living skills .

Anxiety disorders

A. Drahota
Department of Psychiatry, University of California, San Diego,
9500 Gilman Drive (MC: 0603), La Jolla, CA 92093-0603, USA

A. Drahota $(\square)$

Child and Adolescent Services Research Center, Rady

Children's Hospital, 3020 Children's Way, MC 5033, San

Diego, CA 92123, USA

e-mail: adrahota@ucsd.edu

J. J. Wood · K. M. Sze · M. Van Dyke

Graduate School of Education and Information Studies,

University of California, Los Angeles, Moore Hall, Box 951521,

405 Hilgard Ave., Los Angeles, CA 90095-1521, USA

\section{Introduction}

Autism spectrum disorders (ASD) are pervasive and chronic neurobiological disorders characterized by a pattern of impairments in social reciprocity, communication, and behavioral flexibility that permeates the individual's behavior across situations (American Psychiatric Association 2000). In addition to these impairments, up to $80 \%$ of children with high-functioning ASD have one or more concurrent anxiety disorders (de Bruin et al. 2007), making high anxiety a common feature in this population. Impaired self-help skills are common in children with ASD and contribute to overall high levels of morbidity seen in ASD throughout the lifespan (Howlin et al. 2004). Emerging research also suggests a link between anxiety disorders and impairments in daily living skills (Drahota et al. 2010). Children with both ASD and anxiety disorders are thus at high risk of poorly developed daily living skills, warranting intervention in this domain.

Researchers have recently proposed that parental overinvolvement in their children's basic self-care tasks plays a key role in the maintenance of childhood anxiety (Wood 2006). This model suggests that parental over-involvement in basic self-care tasks limit their children's mastery of these tasks, facilitating dependence and overreliance on parents, which maintains feelings of anxiety. Therefore, changes in children's daily living skills should correlate with changes in children's anxiety symptom severity.

Development of interventions targeting the daily living skill deficits of children with autism has been of interest for some time (Kanner 1943). Various interventions have been developed to ameliorate symptoms of ASD and improve communication and social skills of children with ASD (e.g., applied behavior analysis, pivotal response training). Yet, no interventions currently exist which are considered 
efficacious for improving daily living skills for children with high-functioning ASD (i.e., children with ASD having an IQ at or above 70 ).

Daily living skills are developmentally appropriate practical skills necessary to care for oneself and meet daily challenges, consisting of personal skills (ranging from dressing oneself to avoiding sick people in order to remain healthy), home or school skills (ranging from putting things away with reminders to cleaning with cleaning products), and community skills (ranging from knowing it is unsafe to accept rides from strangers to telling the time) (Sparrow et al. 1984). Daily living skills are both currently mastered and performed regularly by children; yet, children with ASD often do not perform skills even if they are capable of doing so. A child's mastery of daily living skills contributes strongly to prognosis (Gillham et al. 2000) and have been noted as "essential" to an individual's ability to function successfully and independently in the world (Liss et al. 2001). However, many parents report difficulty teaching daily living skills to children with ASD, finding it easier to perform such tasks for their child (e.g., Koegel and Egel 1979). It may be that due to low motivation and limited perspective-taking (e.g., for social norms regarding developmentally appropriate independent behaviors, such as when peers are dressing or bathing without parental assistance), children with ASD rarely defy their parents' efforts to do these tasks for them.

Family-based cognitive behavioral therapy (CBT) is a probably efficacious intervention for the treatment of anxiety disorders in typically developing children and children with ASD (e.g., Wood et al. 2009; Wood et al. 2006) and may improve daily living skills in typically developing populations as well (Flay et al. 2005). CBT is a treatment paradigm in which new skills are developed by motivating children and parents with logical and persuasive rationales and Socratic questioning, challenging irrational cognitions maintaining maladaptive and avoidant behaviors, and practicing these skills hierarchically in real-life (in vivo) situations. Given the importance of daily living skills within the ASD population, developing efficacious interventions to improve functioning is a high priority. Accordingly, this study investigated the effects of the Wood et al. (2009) family-based CBT program on parental perceptions of daily living skills of children with ASD and concurrent anxiety disorders.

\section{Method}

\section{Participants}

This study utilizes a sample of children with ASD referred by a medical center-based autism clinic, regional centers, parent support groups, and school personnel for treatment of comorbid anxiety disorders (see Wood et al. (2009) for a detailed description of the sample, the treatment manual, and treatment outcome for child anxiety symptoms). The intent-to-treat sample included 40 children with ASD and an anxiety disorder living in a major metropolitan area of the western United States, ranging in age from 7 to 11 years $(M=9.20, \mathrm{SD}=1.49)$, and their primary parent (defined as parents primarily responsible for overseeing the child's daily activities). Sample size was determined using a power analysis assuming a large effect size (ES) for group differences at posttreatment/postwaitlist (see Wood et al. 2009). With an expected sample of 40 children, and assuming a treatment effect size of .8 standard deviations for all anxiety measures, the power to detect a significant effect for time was $.97(p<.05)$.

Participants met the following inclusion criteria: (a) met research criteria for a diagnosis of autism, Asperger syndrome, or PDD-NOS; (b) met research criteria for one of the following: separation anxiety disorder, social phobia, or obsessive compulsive disorder; (c) were not taking any psychiatric medication at the baseline assessment, or were taking a stable dose of psychiatric medication (i.e., at least one month at the same dosage prior to the baseline assessment), and (d) if medication was being used, children maintained the same dosage throughout the study. Families were excluded if (a) the child had a verbal IQ less than 70 (as assessed in previous testing, or, if there was any question about the child's verbal abilities noted by the independent evaluator at baseline, on the basis of the Wechsler Intelligence Scale for Children-IV administered by the independent evaluator); (b) the child was currently in psychotherapy or social skills training, or was receiving behavioral interventions such as applied behavior analysis, (c) the family was currently in family therapy or a parenting class, (d) the child began taking psychiatric medication or changed his/her dosage during the intervention, or (e) for any reason the child or parents appeared unable to participate in the intervention program.

Table 1 presents descriptive information for participating families. Thirty-seven primary parents also reported their annual family income. Nine (24.3\%) reported an income below $\$ 40,000 ; 10(27.1 \%)$ reported an income between $\$ 40,001$ and $\$ 90,000$; and $18(48.6 \%)$ reported an income over $\$ 90,000$ per year.

Intervention Program

Therapists included 11 doctoral students in clinical or educational psychology and two doctoral-level psychologists. All therapists were in (or had graduated from) an educational or clinical psychology doctoral program at a major research university, had at least 1 year of previous clinical 
Table 1 Descriptive and diagnostic information on families in the IT and WL intervention groups

\begin{tabular}{lllr}
\hline & IT condition No. $(\%)$ & WL condition No. $(\%)$ & $X^{2} / t$ test \\
\hline Intervention completers & $14(82 \%)$ & $22(96 \%)$ & $15(65 \%)$ \\
Child sex (male) & $12(71 \%)$ & $9.22(S D=1.57)$ & $.13, n s$ \\
Child age & $9.18(S D=1.42)$ & $18(78 \%)$ & $-.09, n s$ \\
Primary parent sex (female) & $14(82 \%)$ & $13(60 \%)$ & $.10, n s$ \\
Parent graduated college & $12(71 \%)$ & $11(48 \%)$ \\
Child ethnic background & & $3(13 \%)$ \\
Caucasian & $8(47 \%)$ & $2(9 \%)$ \\
Latino/Latina & $2(12 \%)$ & $1(4 \%)$ \\
Asian & $4(23 \%)$ & $6(26 \%)$ \\
African American & 0 & $11(48 \%)$ \\
Multiracial/other & $3(18 \%)$ & $11(48 \%)$ \\
Autism spectrum disorders & & $1(4 \%)$ \\
Autistic disorder & $9(53 \%)$ & $2.50, n s$ \\
PDD-NOS & $6(35 \%)$ & $1.13, n s$ \\
Asperger syndrome & $2(12 \%)$ & \\
\hline
\end{tabular}

Note. None of the group differences were statistically significant $(n s=$ not statistically significant). IT $=$ immediate treatment condition; $\mathrm{WL}=3$-month waitlist condition

experience, and experience working with children with autism. Therapists received 8 hours of initial training on the intervention, read the treatment manual, listened to a full set of audiotapes of a model therapist conducting the treatment with a pilot study participant, and attended weekly meetings with a clinical supervisor (doctoral level psychologists who developed the protocols). Therapists worked with families for 16 weekly sessions, each lasting $90 \mathrm{~min}$ (about $30 \mathrm{~min}$ with the child and 60 min with the parents/family), implementing the Building Confidence CBT program (Wood and McLeod 2008) modified for use with children with ASD (Wood et al. 2007). As with other CBT programs, the manual includes coping skills training (e.g., affect recognition, cognitive restructuring, and the principle of exposure) followed by in vivo practice of the skills.

Modifications to the manual were designed to address poor adaptive skills deficits, social skills deficits, circumscribed interests and stereotypies, poor attention and motivation, common comorbidities in ASD (e.g., disruptive behavior disorders), and school-based problems. Of particular note for the current study, the Encouraging Independence component of treatment addresses parent and child motivation to increase children's daily living skills acquisition and use. This module is usually presented in the second session of treatment, with ongoing follow-up throughout the treatment to ensure continued progress. In this portion of the treatment, therapists highlight to parents the necessity of focusing attention and effort on developing daily living skills in order to build a foundation for future development and success. With children, therapists work to re-conceptualize the idea of performing new daily living skills independently (cognitive restructuring), (a) highlighting children's increasing responsibility and self-efficacy as a result of performing the skills independently, (b) focusing on social norms-that is, what is developmentally appropriate for the child to be doing without assistance, and (c) providing positive regard when a small step is attempted and mastered. Through discussion and questioning, therapists and children identify maladaptive cognitions (e.g., "I can't do this because if I do, my mom won't help me with anything ever again."), question the validity of the identified cognitions ("Would she really not help with anything else?"), and restructure or correct the cognitive distortions and deficiencies ("Even if I do this myself, my mom will probably help me with the things that I still need help with.”). A plan for building up children's daily living skills hierarchically is developed (gradual exposure). Parents are trained to support their children's attempts at independence through communication skills such as giving choices, immediate positive feedback, and reinforcement strategies.

Trained graduate student independent evaluators blind to the intervention condition of each family conducted diagnostic interviews, assessing constructs such as anxiety disorders and daily living skills, before and immediately after intervention or waitlist and then at 3-month follow-up for the immediate treatment group.

Anxiety Diagnosis Interview Schedule

Children's Diagnostic and Statistical Manual of Mental Disorders (DSM-IV) (American Psychiatric Association 
2000) disorders were assessed on the basis of separate semi-structured diagnostic interviews with the caregiver(s) and the child using the ADIS-C/P (Silverman and Albano 1996), an instrument with favorable psychometric properties (e.g., Wood et al. 2002). Positive reports from either parent or child (the "or" rule) were considered sufficient for rating a criterion as present (Piacentini et al. 1992). Evaluators made ratings on the ADIS-C/P Clinical Severity Rating scale $(\mathrm{CSR} ; 0=$ not at all, $4=$ some, $8=$ very, very mисh) for each assigned diagnosis. Ratings of 4 or above are considered to be of a clinical level.

\section{Daily Living Skills Measures}

Children's daily living skills were assessed on the basis of semi-structured clinical interviews with the caregiver(s) using the Vineland Adaptive Behavior Scale (VABS; Sparrow et al. 1984) and the parent-reported Parent-Child Interaction Questionnaire (Wood 2006). The VABS Survey Form (Sparrow et al. 1984) is a valid and reliable assessment of adaptive behavior comprised of 297 items, reflecting developmental skills in five clinically and empirically derived domains: communication, daily living skills, socialization, motor skills, and maladaptive behavior. For this study, the daily living skills domain of the VABS was used, which is subdivided into personal, community, and family daily living skills. The daily living skills has been age- and gender-normed. Parents report whether their child "Yes, usually" (score of 2), "Sometimes or partially" (score of 1), or "No, never" (score of 0 ) independently performs each activity (e.g., "Washes and dries face without assistance", "Bathes or showers without assistance") which comprises the daily living skills domain.

The Parent-Child Interaction Questionnaire (PCIQ; Wood 2006) addresses concrete, observable parent-child interactions that have occurred during a 1-week time frame using a rating scale based on the frequency of each behavior: 1 (This never or almost never occurred), 2 (This sometimes occurred), or 3 (This almost always occurred). The questionnaire contains 35 items; eight comprise the parent intrusiveness subscale (Wood 2006) used in this study. Items on this subscale focus on parental help with children's private daily routines that most children above age 5 years are capable of performing independently (e.g., dressing, bathing), intrusions on children's personal space (lying with child on child's bed at night), and infantilizing behavior (e.g., using baby words). Cronbach's alpha coefficient for the parental intrusiveness subscale was acceptable (.76).

\section{Procedure}

This study was conducted in compliance with a universitybased IRB. Phone contact was initiated by parents referred to the study. Parents gave written consent and children gave written assent to participate in the study. Baseline diagnostic interviews and pencil-and-paper measures were completed over the course of two days. Children who met all inclusion/exclusion criteria were block randomized by a research assistant to either immediate treatment (IT) or waitlist (WL) using a computer randomization program. Block randomization procedures stratified children based on age and gender. Therapists were randomly assigned to children's cases. Posttreatment assessments were completed on the final day of treatment or within a week of termination; postwaitlist assessments were conducted three months after the baseline assessment but before initiating CBT. Follow-up occurred for IT families three months after termination. These posttreatment, postwaitlist and follow-up assessments involved re-administering all of the outcome measures. Treatment was provided in a research setting, with the exception of the two meetings held at the child's school. Families were offered $\$ 20$ for completing the assessments.

\section{Results}

Means and standard deviations for each group at intake and posttreatment/postwaitlist assessments can be found in Table 2. Intent-to-treat (ITT) analyses of the sample $(N=40)$ were conducted. For the four children who dropped out (3 for IT and 1 for WL, respectively), baseline scores were carried forward to posttreatment/postwaitlist, allowing for a conservative analysis of treatment efficacy. No statistical differences were found on pretreatment demographic variables or baseline VABS and PCIQ measures (see Table 2).

\section{Daily Living Skills}

To examine group differences in total Daily Living Skills as well as Personal Daily Living Skills, an analysis of covariance (ANCOVA) was conducted with baseline VABS raw scores as the covariate, group (e.g., IT versus $\mathrm{WL})$ as the independent variable, and posttreatment/postwaitlist VABS raw scores as the dependent variable. For the VABS total Daily Living Skills, a significant effect was found for group, $F(1,37)=4.06, p=.05$, indicating a significant difference between groups at posttreatment/ postwaitlist.

Raw scores were converted into standard scores and age equivalency scores (Table 2) for better clinical use and comprehension. For the IT group, the mean standard score increased from 50.06 to 60.24 , whereas the mean standard score for children in the WL condition did not change (55.61 at intake; 55.62 at postwaitlist). Age equivalency 
Table 2 Raw score means, standard deviations ${ }^{\mathrm{c}}$, standard scores, and age equivalencies for total and personal daily living skills, and PCIQ parental involvement by group and assessment point

\begin{tabular}{|c|c|c|c|c|c|}
\hline \multirow[t]{2}{*}{ Measure } & \multicolumn{2}{|l|}{ Intake } & \multicolumn{2}{|l|}{ Post ${ }^{\mathrm{b}, \mathrm{c}}$} & \multirow{2}{*}{$\frac{\text { Follow-up }}{\text { IT }}$} \\
\hline & IT & WL & IT & WL & \\
\hline \multicolumn{6}{|l|}{ VABS—total daily living skills } \\
\hline Raw score mean $(S D / S E)^{\mathrm{c}}$ & $93.47(29.91)$ & $97.43(23.91)$ & $109.63(4.07)$ & $98.80(3.50)$ & 114.24 \\
\hline Mean standard score & 50.06 & 55.61 & 60.24 & 55.62 & 70.00 \\
\hline Mean age equivalency & 5.2 years & 5.4 years & 6.0 years & 5.7 years & 6.7 years \\
\hline \multicolumn{6}{|c|}{ VABS—personal daily living skills ${ }^{\mathrm{a}}$} \\
\hline Raw score mean $(S D / S E)^{\mathrm{c}}$ & $55.54(10.85)$ & $57.49(9.27)$ & $62.81(1.54)$ & $58.30(1.32)$ & $63.65(9.33)$ \\
\hline Mean age equivalency & 4.1 years & 4.5 years & 5.0 years & 4.6 years & 5.2 years \\
\hline \multicolumn{6}{|l|}{ PCIQ_parental involvement } \\
\hline Raw score mean $(S D / S E)^{\mathrm{c}}$ & $13.53(3.78)$ & $14.30(3.78)$ & $11.93(.55)$ & $13.53(.48)$ & $10.89(2.93)$ \\
\hline
\end{tabular}

Note. $\mathrm{IT}=$ immediate treatment condition; $\mathrm{WL}=3$-month waitlist condition. VABS $=$ Vineland Adaptive Behavior Scale; PCIQ $=$ ParentChild Interaction Questionnaire

${ }^{\text {a }}$ For the VABS-Total Daily Living Skills, standard score mean is $100(S D=15)$

b The raw score mean at posttreatment/postwaitlist is adjusted for pretreatment scores

c Standard errors, accounting for the pretreatment score adjustment, are reported at posttreatment/postwaitlist rather than standard deviations

scores were determined from the unadjusted mean scores from both groups at intake and posttreatment/postwaitlist. The total Daily Living Skills of children in the IT condition averaged 5.2 years at intake and increased to 6.0 years at posttreatment. Children in the WL condition averaged 5.4 years at intake and increased to 5.7 years at postwaitlist. Clearly, the majority of children in both groups remained delayed, but children in the IT condition improved by a half of a standard deviation on the mean standard score and almost a full year in the age equivalency scores, whereas children in the WL condition did not improve on the mean standard score and increased only three months in age equivalency.

For the VABS Personal Daily Living Skills raw scores, a significant effect was also found for group $F$ (1, $37)=4.93, p<.05$. Age equivalency scores were derived. Children in the IT group increased their Personal Daily Living Skills from 4.1 to 5.0 years, while children in the WL group only increased their scores from 4.5 to 4.6 years. While substantially lower than their chronological age, children in the IT group advanced in Personal Daily Living Skills by almost a full year over the course of the intervention whereas children in the WL group only advanced by 1 month, indicating that the mere passage of time did not facilitate the improvement.

Posttreatment effect sizes (ES) for VABS total Daily Living Skills scores and Personal Daily Living Skills scores were .45 , a small effect, and .50 , a medium effect, respectively (Cohen 1988). Paired $t$ tests indicate that there were significant changes from pretreatment to posttreatment in parent-reported VABS total and Personal Daily Living Skills for the IT condition $(t(16)=-3.72, p<.01$; $t(16)=-3.82, p<.01)$; as expected, this was not found for the WL condition $(t(22)=-.72, p=.48 ; t(22)=-1.21$, $p=.24)$. Children in the IT group made significantly greater gains in total VABS Daily Living Skills scores from pretreatment to posttreatment than children in the WL group as indicated by within-group effect size calculations $(\mathrm{ES}=.48$, a small effect, \& .11, a trivial effect, respectively), and in VABS Personal Daily Living Skills scores $(\mathrm{ES}=.58$, a medium effect, and .16 , a minimal effect, respectively).

\section{Parental Intrusiveness}

To examine group differences in PCIQ parental intrusiveness scores, an ANCOVA was conducted with baseline PCIQ raw score as the covariate, group (e.g., IT versus WL) as the independent variable, and posttreatment/postwaitlist PCIQ raw scores as the dependent variable. A statistically significant difference between the IT and WL groups at posttreatment/postwaitlist was found, $F(1,37)=4.75, p<.05$. Unnecessary parental involvement PCIQ scores were lower at posttreatment/ postwaitlist for children in IT as compared to WL (ES $=.59$, a medium effect). Parents of children in the IT group made significantly greater reductions in parental involvement in their children's personal self-care tasks from pretreatment to posttreatment than parents of children in the WL group as indicated by within-group effect size calculations ( $E S=.51$, a medium effect, and .14 , a trivial effect, respectively) and paired $t$ test, $(t(16)=3.16, \quad p<.01 ; \quad t(22)=1.09, \quad p=.29$, respectively). 
Maintenance of Treatment Gains at 3-Month Follow-up

In the IT condition, 10 families $(71.4 \%$ of the treatment completers) returned for a 3-month follow-up assessment (Table 2); no statistical differences were found between children who did or did not return for the follow-up assessment on demographics or measures of interest. Results from within-subjects $t$ tests, comparing parentreported VABS total and Personal Daily Living Skills, and PCIQ parental intrusiveness at posttreatment and follow-up indicate that the treatment effect was maintained through the follow-up period: $t(8)=-.38, p=.72 ; t(8)=-.05$, $p=.97 ;$ and $t(8)=.21, p=.84$, respectively.

\section{Correlational Analyses Between Change in Anxiety} and Daily Living Skills Scores

Bivariate correlational analyses examined the relation between change in anxiety scores and change in outcome measures (Table 3). Change in anxiety scores were calculated by subtracting the ADIS clinician severity score of the primary anxiety disorder at intake from the ADIS clinician severity score of the primary anxiety disorder at posttreatment/postwaitlist. Intake scores for each of the outcome questionnaires (e.g., VABS total and personal DLS, PCIQ parent intrusiveness) were subtracted from posttreatment/postwaitlist scores to calculate change scores. Negative scores on all measures indicate improvements. Correlational analyses suggest an association between the ADIS clinician severity score change and parent-reported independent daily living skills and parental intrusiveness.

\section{Discussion}

The present study provides initial evidence that CBT may increase parental perceptions of daily living skills in children with ASD and concurrent anxiety disorders, and

Table 3 Correlational analyses between anxiety and daily living skills change scores by group

\begin{tabular}{llll}
\hline & \multicolumn{4}{l}{ ADIS clinician severity rating-change score } \\
\cline { 2 - 4 } & Total sample $(n=40)$ & IT $(n=17)$ & WL $(n=23)$ \\
\hline Total DLS & $.57(p<.001)$ & $.49(p<.05)$ & $.56(p<.01)$ \\
Personal DLS & $.49(p<.001)$ & $.33(p=.20)$ & $.43(p<.05)$ \\
PCIQ & $.39(p<.05)$ & $.41(p=.11)$ & $.13(p=.56)$ \\
\hline
\end{tabular}

Note. ADIS = Anxiety Disorders Interview Schedule-IV Parent and Child versions; IT $=$ immediate treatment condition; $\mathrm{WL}=$ waitlist condition; Total DLS = VABS Total Daily Living Skills; Personal DLS $=$ VABS Personal Daily Living Skills; PCIQ = Parent-Child Interaction Questionnaire - Parental Intrusiveness scale decrease corresponding unnecessary parental involvement in their children's personal self-care tasks. Furthermore, gains in daily living skills for a subgroup of children who received treatment were maintained over 3 months. The CBT intervention techniques employed may promote adaptive skill acquisition among children while encouraging parents to support their children's skill use and grant opportunities to become more self-sufficient. Conversely, other factors may have affected these outcomes, such as parental reporting bias. Additionally, further study of the efficacious components of CBT is warranted. That is, behavioral therapy alone may prove equally efficacious. Nonetheless, the results of this study could have theoretical as well as practical implications.

As hypothesized, parents of children in the IT condition perceived an increase in personal daily living skills regularly performed by children, such as grooming, toileting, dressing, and simple healthcare, as compared with children in the WL group. However, although statistically significant, we must consider whether the gain is clinically significant. The standard and age-equivalency scores indicate that children in the IT condition continued to fall well below the expected total and personal daily living skills range for their age group at posttreatment. It is unclear whether more intervention emphasis on daily living skills within the current intervention framework (i.e., devoting a higher proportion of each session to helping children build skills and training parents how to support independence) is needed to affect greater improvements or whether the current intervention would produce additional gains if it were longer in duration. Due to the pervasive and chronic pattern of adaptive functioning impairments in social reciprocity, communication, and flexibility with behavior and activities, ASD may cause children to "hit a wall" in their development of daily living skills. Without even more extensive external support and guidance, children with ASD and their parents may find it challenging to develop daily living skills that match children's chronological age. On the other hand, naturalistic studies of children with autism show little to no gains in adaptive behavior, including daily living skills, over similar timeframes (Smith et al. 2000), indicating that any improvement may be meaningful.

Consistently, studies investigating the adaptive functioning and daily living skills of individuals with ASD have found great variability in skills (Howlin et al. 2004; Howlin et al. 2000; Liss et al. 2001); however, the overwhelming majority of individuals with ASD are reported to have "poor" outcomes, including little independence from caregivers. Similarly, the present study found variability in parental perceptions of their children's total and personal daily living skills at intake. This variability continued through the intervention for children in the immediate 
treatment and waitlist conditions. This pattern of findings suggests that additional variables may contribute to the maintenance of impaired daily living skills. Age, cognitive scores, symptom severity of autism, and memory have been associated with the adaptive functioning and daily living skills of children with ASD. For example, Freeman et al. (1999) found that adaptive functioning increased with age for individuals with ASD and was related to individual initial cognitive scores. Liss et al. (2001) and Baghdadli et al. (2007) found severity of autistic symptoms related to poorer adaptive functioning outcomes, suggesting that symptoms of autism may interfere with the development of daily living skills. Finally, poorer memory has been related with poor adaptive functioning skills (Liss et al. 2001). Future studies should investigate the role of these child factors in determining the efficacy of the CBT intervention involving subgroups of children with ASD.

In addition, parent motivation, beliefs about treatment credibility and expectancies, and treatment adherence may play a role in the variance of Daily Living Skills scores at posttreatment. CBT requires parents play an active role in treatment implementation; parents are asked to use treatment strategies independently, regularly, and correctly. Studies of parent beliefs find that treatment credibility is significantly associated with parental motivation to perform treatment techniques, while parental expectations of treatment outcome predict parental treatment adherence (Nock et al. 2007). Therefore, variability in children's daily living skills may partially be a function of parents' motivation and beliefs. Research regarding these factors may provide additional details regarding the variable treatment response despite seemingly efficacious intervention techniques.

Recently, the Research Units on Pediatric Psychopharmacology [RUPP] Autism Network (2007) reported on the feasibility of a manualized parent training program involving parents of children with ASD in which children's noncompliance behaviors and daily living skills were targets of intervention. Parents attended and adhered to the parent training intervention, and children were reported to decrease noncompliance and increase their daily living skills, as reported on the parent-reported VABS Daily Living Skills subscale, by $22.3 \%$ over the entirety of the intervention. While this study did not include a control group, it provides additional support for parental training as essential for facilitating independence in children with ASD.

Finally, parents of children in the immediate treatment condition reported that their own involvement in their child's private daily self-care tasks significantly decreased when compared with parents of children in the waitlist condition. A medium effect size was found when comparing pretreatment and posttreatment parental involvement scores for parents whose children received treatment, while a negligible effect was found for parents of children in the WL group. This is an important contribution to the treatment literature because parental behaviors, such as involvement in their children's basic self-care tasks, have been implicated as a factor in the maintenance of anxiety and impaired daily living skills (Wood 2006). According to Wood's model of parental intrusiveness, when caregivers take over tasks that children could be performing independently, they limit children's mastery and facilitate dependence, maintaining anxiety. Alternatively, children with daily living skill deficits may become anxious due to their excessive reliance on caregivers for assistance with tasks that peers are capable of performing independently. Therefore, decreases in parental intrusiveness in their children's personal self-care tasks likely facilitate independence and may assist in skill acquisition and mastery, as well as potentially lower anxiety levels.

In fact, a correlation was found between changes in anxiety severity and daily living change scores, indicating some relation between these constructs. In particular, these results may indicate that possibility of a mediating effect of daily living skills. That is, increasing daily living skills may be related to a reduction in anxiety severity. Interestingly, when examining the correlation strength, correlations are not stronger in the IT versus WL group, which would be expected in a true mediation relationship. Overall, however, these results indicate that if there is a change in anxiety severity then daily living skills are also likely to change. Only further testing will determine whether a mediating relationship exists and the nature of the relation.

It is particularly notable that parent perceptions of treatment gains resulting from the CBT intervention remained stable over time without continued intervention. Parent reports suggested the maintenance of their reduced involvement with their children's private self-care routines such as dressing and bathing, intrusions on children's personal space, and infantilizing behaviors. These findings are provocative because of the difficulty sustaining treatment gains across time and settings when involving children with ASD (Lord et al. 2005). This maintenance may be due to several factors. During treatment, therapists do not continue to progress onto new and more complex daily living skills until the previous skill or step is mastered and generalization is evident. Thus, skills are developed and performed reliably, increasing the likelihood that they will be maintained. Even if children do not become fully selfmotivating, parents are likely to continue adhering to treatment techniques due to their own observation of their children's abilities.

Limitations

Parent reports were used to determine the children's daily living skills as well as their own involvement in their 
child's personal self-care tasks at intake and posttreatment/ postwaitlist. Because parents are aware of the treatment condition to which they are assigned, parent reports could have been influenced by a desire to provide responses consistent with treatment goals. However, childhood anxiety disorders were the main focus of the intervention and parent's primary reason to seek treatment; most of the questionnaires completed by parents focused on anxiety disorders and related interference. Therefore, questionnaires and semi-structured interviews related to daily living skills may not have been the primary construct considered during assessments by parents and thus may have been more accurately reported. Future studies would benefit from multiple informants reporting on children's daily living skills.

Of particular importance is the discussion of generalization of the intervention outcomes across varying families with children with ASD and anxiety. Our sample was slightly skewed towards including families in middle and upper middle class income (18 families reported earning $\$ 90,000$ a year or more), with approximately $60 \%$ of the primary caregivers having completed college. However, ANCOVA was used to analyze group differences in posttreatment VABS total and Personal Daily Living Skills and PCIQ parental intrusiveness across the three income groups (less than $\$ 40,000 ; \$ 40,001-\$ 89,999$; more than $\$ 90,000$ ), with pretreatment VABS total and Personal Daily Living Skills and PCIQ scores as covariates. Results indicate these analyses were not significant ( $p$ 's ranged from .26 to .94, respectively).

\section{Implications and Conclusions}

Family CBT techniques appear to produce statistically and (potentially) clinically significant gains in daily living skills among children with ASD. Parents perceived that their children with ASD who received treatment were able to perform more total and personal daily living skills independently at the end of treatment. Facilitating the acquisition of age-appropriate daily living skills early in life may contribute to a trajectory promoting more opportunities for independent functioning as children develop. Parents who received treatment reported that they reduced their intrusions in their children's private self-care tasks, which may give them more time for other activities and allow them to disengage in small ways from their children's moment-to-moment functioning. With its focus on taking small steps and emphasizing each accomplishment, the CBT model appears well suited for supporting ongoing growth. In future research on daily living skills interventions for ASD, it will be important to determine if parents and children are able to maintain gains over the course of years, not just months, if a focus on the importance of this domain of functioning in CBT might lead to an acceleration in growth that eventually helps children and youth attain age-appropriate levels of independence, and how mastering daily living skills relates to ameliorating anxiety disorder symptoms or severity.

Acknowledgments This study was supported by National Institute of Mental Health grants F31-MH-73213 (PI: Amy Drahota) and R03MH-075806 (PI: Jeffrey J. Wood), the Cure Autism Now Foundation (PI: Jeffrey J. Wood), and the UCLA Center for Autism Research and Training (PI: Jeffrey J. Wood). There is no conflict of interest affecting the study authors with regard to this study.

Open Access This article is distributed under the terms of the Creative Commons Attribution Noncommercial License which permits any noncommercial use, distribution, and reproduction in any medium, provided the original author(s) and source are credited.

\section{References}

American Psychiatric Association. (2000). Diagnostic and statistical manual of mental disorders. Text Revision (DSM-IV TR) (4th ed. ed.). Washington, DC: American Psychiatric Association.

Baghdadli, A., Picot, M.-C., Michelon, C., Bodet, J., Pernon, E., Burstezjn, C., et al. (2007). What happens to child with PDD when they grow up? Prospective follow-up of 219 children from preschool age to mid-childhood. Acta Psychiatrica Scandinavica, 115, 403-412.

Cohen, J. (1988). Set correlation and contingency tables. Applied Psychological Measurement, 12, 425-434.

de Bruin, E. I., Ferdinand, R. F., Meester, S., de Nijs, P. F. A., \& Verheij, F. (2007). High rates of psychiatric co-morbidity in PDD-NOS. Journal of Autism and Developmental Disorders, 37, 877-886.

Drahota, A., Wood, J. J., Hwang, W-C., Langley, A., Piacentini, J. C. (2010). Adaptive functioning in school-aged children: Linkages with Trait Anxiety and Anxiety Disorders (in submission).

Flay, B. R., Biglan, A., Boruch, R. F., Gonzalez Castro, F., Gottfredson, D., Kellam, S., et al. (2005). Standards of evidence: Criteria for efficacy, effectiveness and dissemination. Prevention Science, 6, 151-175.

Freeman, B. J., Del' Homme, M., Guthrie, D., \& Zhang, F. (1999). Vineland adaptive behavioral scale scores as a function of age and initial IQ in 210 autistic children. Journal of Autism and Developmental Disorders, 29, 379-384.

Gillham, J. E., Carter, A. S., Volkmar, F. R., \& Sparrow, S. S. (2000). Toward a developmental operational definition of autism. Journal of Autism and Developmental Disorders, 30, 269-278.

Howlin, P., Goode, S., Hutton, J., \& Rutter, M. (2004). Adult outcome for children with autism. Journal of Child Psychology and Psychiatry, 45, 212-229.

Howlin, P., Mawhood, L., \& Rutter, M. (2000). Autism and developmental receptive language disorder-A follow-up comparison in early adult life. II: Social, behavioural, and psychiatric outcomes. Journal of Child Psychology and Psychiatry, 41, 561578.

Kanner, L. (1943). Autistic disorders of affective contact. Nervous Child, 2, 217-250.

Koegel, R. L., \& Egel, A. L. (1979). Motivating autistic children. Journal of Abnormal Psychology, 88, 418-426.

Liss, M., Harel, B., Fein, D., Allen, D., Dunn, M., Feinstein, C., et al. (2001). Predictors and correlates of adaptive functioning in 
children with developmental disorders. Journal of Autism and Developmental Disorders, 31, 219-230.

Lord, C., Wagner, A., Rogers, S., Szatmari, P., Aman, M., Charman, T., et al. (2005). Challenges in evaluating psychosocial interventions for autistic spectrum disorders. Journal of Autism and Developmental Disorders, 35, 695-708.

Nock, M. K., Ferriter, C., \& Holmberg, E. (2007). Parent beliefs about treatment credibility and effectiveness: Assessment and relation to subsequent treatment participation. Journal of Child and Family Studies, 16, 27-38.

Piacentini, J. C., Cohen, P., \& Cohen, J. (1992). Combining discrepant diagnostic information from multiple sources: Are complex algorithms better than simple ones? Journal of Abnormal Child Psychology, 20, 51-63.

Research Units on Pediatric Psychopharmacology [RUPP] Autism Network. (2007). Parent training for children with pervasive developmental disorders: A multi-site feasibility trial. Behavioral Interventions, 22, 179-199.

Silverman, W. K., \& Albano, A. M. (1996). The Anxiety Disorders Interview Schedule for DSM-IV: Child and Parent Versions. San Antonio, TX: Psychological Corporation.

Smith, T., Groen, A. D., \& Wynn, J. W. (2000). Randomized trial of intensive early intervention for children with pervasive developmental disorder. American Journal on Mental Retardation, 105, 269-285.
Sparrow, S., Balla, D., \& Cicchetti, D. (1984). Vineland scales of adaptive behavior: Survey form manual. Circle Pines, MN: American Guidance Service.

Wood, J. J. (2006). Parental intrusiveness and children's separation anxiety in a clinical sample. Child Psychiatry and Human Development, 37, 73-87.

Wood, J. J., Drahota, A., \& Sze, K. M. (2007). Cognitive behavioral therapy for anxiety and social problems in children with autism: Intervention manual. Los Angeles: Unpublished intervention manual developed at the University of California.

Wood, J. J., Drahota, A., Sze, K., Har, K., Chiu, A., \& Langer, D. A. (2009). Cognitive behavioral therapy for anxiety in children with autism spectrum disorders: A randomized, controlled trial. Journal of Child Psychology and Psychiatry, 50, 224-234.

Wood, J. J., \& McLeod, B. (2008). Child anxiety disorders: A treatment manual for practitioners. New York: Norton.

Wood, J. J., Piacentini, J. C., Bergman, R. L., McCracken, J., \& Barrios, V. (2002). Concurrent validity of the anxiety disorders section of the Anxiety Disorders Interview Schedule for DSMIV: Child and parent versions. Journal of Clinical Child \& Adolescent Psychology, 31, 335-342.

Wood, J. J., Piacentini, J. C., Southam-Gerow, M., Chu, B. C., \& Sigman, M. (2006). Family cognitive behavioral therapy for child anxiety disorders. Journal of the American Academy of Child and Adolescent Psychiatry, 45, 314-321. 